\title{
External Fractional-Order Gradient Vector Perona-Malik Diffusion for Sinogram Restoration of Low-Dosed X-Ray Computed Tomography
}

\author{
Shaoxiang Hu \\ School of Automation Engineering, University of Electronic Science and Technology of China, Chengdu, Sichuan 611731, China \\ Correspondence should be addressed to Shaoxiang Hu; hushaox@126.com
}

Received 11 September 2013; Accepted 20 September 2013

Academic Editor: Ming Li

Copyright (C) 2013 Shaoxiang Hu. This is an open access article distributed under the Creative Commons Attribution License, which permits unrestricted use, distribution, and reproduction in any medium, provided the original work is properly cited.

Existing fractional-order Perona-Malik Diffusion (FOPMD) algorithms are defined as fully spatial fractional-order derivatives (FSFODs). However, we argue that FSFOD is not the best way for diffusion since different parts of spatial derivative play different roles in Perona-Malik diffusion (PMD) and derivative orders should be decided according to their roles. Therefore, we adopt a novel fractional-order diffusion scheme, named external fractional-order gradient vector Perona-Malik diffusion (EFOGV-PMD), by only replacing integer-order derivatives of "external" gradient vector to their fractional-order counterparts while keeping integerorder derivatives of gradient vector for diffusion coefficients since the ability of edge indicator for 1-order derivative is demonstrated both in theory and applications. Here "external" indicates the spatial derivatives except for the derivatives used in diffusion coefficients. In order to demonstrate the power of the proposed scheme, some real sinograms of low-dosed computed tomography (LDCT) are used to compare the different performances. These schemes include PMD, regularized PMD (RPMD), and FOPMD. Experimental results show that the new scheme has good ability in edge preserving, is convergent quickly, has good stability for iteration number, and can avoid artifacts, dark resulting images, and speckle effect.

\section{Introduction}

Since the work of Perona-Malik in 1990, Perona-Malik diffusion (PMD) becomes a popular technique in image denoising and it is defined as [1]

$$
\frac{\partial u(x, y, t)}{\partial t}=\operatorname{div}[c(\|\nabla u(x, y, t)\|) \nabla u(x, y, t)],
$$

where $u(x, y, 0)$ is the initial gray scale image, $u(x, y, t)$ is the smoothed gray scale image at time $t, \nabla$ denotes the gradient, $\operatorname{div}(\cdot)$ is the divergence operator and $c(\cdot)$ is the diffusion coefficient. $c(\cdot)$ controls the rate of diffusion and it is usually chosen as a monotonically decreasing function of the module of the image gradient.

The key idea of PMD is to smooth the homogenous regions with small $\|\nabla u(x, y, t)\|$ while near singularities with big $\|\nabla u(x, y, t)\|$, PMD only smoothes along the perpendicsular direction of the gradient. This idea is based on that the 1-order gradient module can be used as singularities locator.
Thus the order of gradient vector in diffusion coefficients should be kept as 1. It is the start point of EFOGV-PMD proposed in this paper.

In 1992, Catté et al. indicate that PMD is ill posed and they propose a new well-posed method named regularized Perona-Malik Diffusion (RPMD) by modifying the module of the image gradient in diffusion coefficients to the module of the gradient convoluted with a Gaussian kernel [2].

Although PMD methods have been demonstrated to be able to achieve a good trade-off between noise removal and edge preservation, the resulting image in the presence of the noise often suffers from its "staircase" look. In order to eliminate undesired "staircase" effect, high-order PDEs (typically fourth-order PDEs) for image restoration have been introduced in [3-5]. Though these methods can eliminate the staircase effect efficiently, they often leave the image with isolated black and white speckles (so-called "speckle effect") [6]. 
Recently, fractional-order PMD (FOPMD) has been studied in image denoising [6-9]. The fractional derivative can be seen as the generalization of the integer-order derivative [10-13]. FOPMD whose fractional-order is $\alpha, 0 \leq \alpha \leq 2$ is a "natural interpolation" between PMD and fourth-order PDEs. Therefore, it has the benefits of both of PMD and highorder PDEs.

Bai and Feng proposed a FSFOD method for image denoising with Euler-Lagrange equations of a cost functional and using Fourier domain to compute the fractional derivative [6]. The numerical results showed that both of the staircase effect and the speckle effect can be eliminated effectively.

Zhang and Wei develop a class of fractional-order multiscale variational model using G-L definition of fractionalorder derivative and propose an efficient condition of the convergence for the model [7].

Janev et al. propose a new FOPMD scheme by extending classical spatial derivatives to spatial as well as time fractional derivatives based on R-L derivative [8].

We also reported an experimental study used FOPMD for sinogram restoration of low-dosed computed tomography (LDCT) based on fully spatial FOPMD using G-L definition [9]. In [9], we observe that FOPMD experiments with different fractional-orders show different diffusion behaviors, which lead us to study FOPMD further.

All above new schemes adopt ESFOD schmes. Although they have reported on good performance in preserving edges, suppressing staircase, and speckle effects, the resulting images of these FOPMD methods still have some artifacts and speckle effect which hamper them to be used in LDCT imaging.

Minimizing the radiation exposure to patients has been one of the major efforts in modern clinical X-ray CT radiology [14-16]. However, the presentation of strong noise degrades the quality of LDCT images dramatically and decreases the accuracy of diagnosis.

Filtering noise from clinical scans is a challenging task, since these scans contain artifacts and consist of many structures with different shape, size, and contrast, which should be preserved for making correct diagnosis. In addition, LDCT imaging also requires there are not artifacts to arise while denoising because artifacts also increase chances of mistakes and misdiagnosis. Many strategies have been proposed to reduce noises, but less of them discuss how to avoid artifacts $[9,17-27]$.

The artifacts of existing FOPMD methods for resulting images are generated by locating error positions of edges. Thus the resulting images produce some undesired weak edges, which form the artifacts.

The basic reason for locating edge positions falsely is that fractional-order gradient module cannot be used for edge indicator. That is, edge locations can be located as big leaps of 1-order derivatives while fractional-order derivatives do not have this nature. Thus keeping 1-order derivative to be edge indicator of diffusion coefficients is a better choice compared to existing fully spatial FOPMD.

Following above discussion, we adopt a novel fractionalorder diffusion scheme, named external fractional-order gradient vector Perona-Malik Diffusion PMD (EFOGVPMD), by only replacing integer-order derivatives of external gradient vector to their fractional-order counterparts while keeping 1-order derivatives for diffusion coefficients. Here "external" indicates the spatial derivatives except for the derivatives used in diffusion coefficients. Since locations of edges can be detected correctly by our new scheme, FOPMD can preserve edges well and avoid artifacts.

The arrangement of this paper is as follows: In Section 2, the EFOGV-PMD is introduced, and then the the numerical scheme is given in Section 3, the experiment results are shown and discussed in Section 4, and the final part is the conclusions and acknowledgements.

\section{External Fractional-Order Gradient Vector Perona-Malik Diffusion}

The fractional-order derivative can be computed in a number of ways. The most famous of these definitions are the R-L and Grunwald-Letnikov definitions. In this paper, we use G-L definition defined as follows:

$$
D^{\alpha} g(x)=\lim _{h \rightarrow 0^{+}} \frac{\sum_{k \geq 0}(-1)^{k} C_{k}^{\alpha} g(x-k h)}{h^{\alpha}}, \quad \alpha>0,
$$

where $g(x)$ is a real function, $\alpha>0$ is a real number, $C_{k}^{\alpha}=$ $\Gamma(\alpha+1) /[\Gamma(k+1) \Gamma(\alpha-k+1)]$ is the generalized binomial coefficient and $\Gamma(\cdot)$ denotes the Gamma function.

Isotropic diffusion will damage image features such as edges, lines, and textures. To avoid the damage, smoothing has to be adaptively controlled by the amount of smoothing or the direction of smoothing. A classic example of adaptive smoothing is the anisotropic diffusion scheme proposed by Perona and Malik [1], in which the smoothing process is formulated by a partial differential equation (PDE). PMD is formulated on (1).

However, PMD methods suffer from their "staircase" effects. Therefore, FOPMD is proposed to suppress the staircase of PMD.

The fractional-order gradient vector with $\alpha$ order is defined as

$$
\nabla^{\alpha} u(x, y, t)=\left[\nabla_{x}^{\alpha} u(x, y, t), \nabla_{y}^{\alpha} u(x, y, t)\right],
$$

where $\alpha$ is a positive real, $\nabla_{x}^{\alpha} u(x, y, t)$ represents the partial fractional-order derivative of $u(x, y, t)$ with respect to the variable $x$ whose order is $\alpha$, and $\nabla_{y}^{\alpha} u(x, y, t)$ represents the partial fractional-order derivative of $u(x, y, t)$ with respect to the variable $y$ whose order is $\alpha$.

According to [9], FOPMD is defined as

$$
\frac{\partial u(x, y, t)}{\partial t}=\operatorname{div}^{\alpha}\left[c\left(\left\|\nabla^{\alpha} u(x, y, t)\right\|\right) \nabla^{\alpha} u(x, y, t)\right],
$$

where $\operatorname{div}^{\alpha}$ denotes the $\alpha$-order divergence. For the vector $\nabla^{\beta} v=\left[v_{x}^{\beta}, v_{y}^{\beta}\right]$ where $v_{x}^{\beta}, v_{y}^{\beta}$ represent the partial fractionalorder derivative of $u(x, y, t)$ with whose order is $\beta$ respect to the variable $x$ and $y$ respectively, its $\alpha$-order divergence is defined as

$$
\operatorname{div}^{\alpha} \nabla^{\beta} v=v_{x}^{\beta+\alpha}+v_{y}^{\beta+\alpha}
$$


However, FOPMD defined on (4) will produce some artifacts for sinogram restoration of LDCT, which increases the probability of error diagnosis. In order to avoid artifacts produced in sinogram restoration of LDCT using FOPMD, we propose a new diffusion model, named external fractional-order gradient vector of PMD (EFOGV-PMD) where "external" indicates the gradient vector of PMD except for the gradient vector of diffusion coefficients. That is, the orders of derivatives for spatial gradient vectors on different positions are different, the order of gradient vector used in diffusion coefficients is 1 while order of external gradient vector is a positive real $\alpha$.

Therefore, the EFOGV-PMD is given by

$$
\frac{\partial u(x, y, t)}{\partial t}=\operatorname{div}^{\alpha}\left[c(\|\nabla u(x, y, t)\|) \nabla^{\alpha} u(x, y, t)\right]
$$

with the observed image as the initial condition.

When $\alpha=1,(6)$ is precisely the PMD; when $\alpha=2$, (13) is precisely the fourth-order anisotropic diffusion equation. In this paper, we are interested in $0.5 \leq \alpha \leq 1.5$ since Bai and Feng in [6] suggest that $\alpha=1.5$ in their model has the best performance.

\section{The Numerical Scheme}

An image $U$ will be a 2-dimensional matrix of size $N \times$ $N$. In order to get the aim of anisotropic diffusion along different directions and the discrete 1-order gradient $\nabla \mathbf{u}$ is an 8-dimensional vector:

$$
\begin{aligned}
\nabla \mathbf{u}(\mathbf{i}, \mathbf{j})= & \left(\nabla_{0} u(i, j), \nabla_{1} u(i, j), \nabla_{2} u(i, j), \nabla_{3} u(i, j),\right. \\
& \left.\nabla_{4} u(i, j), \nabla_{5} u(i, j), \nabla_{6} u(i, j), \nabla_{7} u(i, j)\right)^{T},
\end{aligned}
$$

where $T$ represents the transpose of the vector and $\nabla u_{k}(i, j)$, $k=0, \ldots, 7$ are defined as

$$
\begin{aligned}
& \nabla_{0} u(i, j)=u(i, j+1)-u(i, j), \\
& \nabla_{1} u(i, j)=u(i-1, j+1)-u(i, j), \\
& \nabla_{2} u(i, j)=u(i-1, j)-u(i, j), \\
& \nabla_{3} u(i, j)=u(i-1, j-1)-u(i, j), \\
& \nabla_{4} u(i, j)=u(i, j-1)-u(i, j), \\
& \nabla_{5} u(i, j)=u(i+1, j-1)-u(i, j), \\
& \nabla_{6} u(i, j)=u(i+1, j)-u(i, j), \\
& \nabla_{7} u(i, j)=u(i+1, j+1)-u(i, j) .
\end{aligned}
$$

Thus

$$
\begin{aligned}
& \nabla^{2 \alpha} \mathbf{u}(\mathbf{i}, \mathbf{j}) \\
& =\left(\nabla_{0}^{2 \alpha} u(i, j), \nabla_{1}^{2 \alpha} u(i, j), \nabla_{2}^{2 \alpha} u(i, j), \nabla_{3}^{2 \alpha} u(i, j),\right. \\
& \left.\quad \nabla_{4}^{2 \alpha} u(i, j), \nabla_{5}^{2 \alpha} u(i, j), \nabla_{6}^{2 \alpha} u(i, j), \nabla_{7}^{2 \alpha} u(i, j)\right)^{T},
\end{aligned}
$$

where $T$ represents the transpose of the vector. From (14) we have

$$
\begin{aligned}
\nabla_{0}^{2 \alpha} u(i, j)= & \sum_{k=0}^{K-1}(-1)^{k} C_{k}^{2 \alpha} u(i, j+k-(K-1)) \\
\nabla_{1}^{2 \alpha} u(i, j)= & \sum_{k=0}^{K-1}(-1)^{k} C_{k}^{2 \alpha} u \\
& \times(i-k+(K-1), j+k-(K-1)) \\
\nabla_{2}^{2 \alpha} u(i, j)= & \sum_{k=0}^{K-1}(-1)^{k} C_{k}^{2 \alpha} u(i-k+(K-1), j) \\
\nabla_{3}^{2 \alpha} u(i, j)= & \sum_{k=0}^{K-1}(-1)^{k} C_{k}^{2 \alpha} u \\
\nabla_{4}^{2 \alpha} u(i, j)= & \sum_{k=0}^{K-1}(-1)^{k} C_{k}^{2 \alpha} u(i, j-k+(K-1)) \\
\nabla_{5}^{2 \alpha} u(i, j)= & \sum_{k=0}^{K-1}(-1)^{k} C_{k}^{2 \alpha} u \\
\nabla_{7}^{2 \alpha} u(i, j)= & \sum_{k=0}^{2 \alpha}(-1)^{k} C_{k}^{2 \alpha} u \\
\nabla_{6} u(i, j)= & \sum_{k=0}^{K-1}(-1)^{k} C_{k}^{2 \alpha} u(i+k-(K-1), j) \\
\left.\nabla^{2 \alpha}+(K-1), j-k+(K-1)\right) &
\end{aligned}
$$

Let

$$
\mathbf{g}=\left(g_{0}, g_{1}, g_{2}, g_{3}, g_{4}, g_{5}, g_{6}, g_{7}\right)^{T},
$$

where $T$ represents the transpose of the vector and $g_{k}, k=$ $0, \ldots, 7$ is defined as

$$
g_{k}=\frac{g\left(\left\|\nabla_{k} u(i, j)\right\|\right)}{\sum_{n=0}^{7} g\left(\left\|\nabla_{n} u(i, j)\right\|\right)}, \quad k=0,1, \ldots, 7,
$$

where $\nabla_{k} u(i, j), k=0, \ldots 7$ defined in (7) are the components of vector $\nabla \mathbf{u}(\mathbf{i}, \mathbf{j}), \sum_{n=0}^{7} g\left(\left\|\nabla_{n} u(i, j)\right\|\right)$ is the normalized constant, and $g$ is the decreasing function of absolute value of $\nabla_{k} u(i, j), k=0, \ldots 7$. In [1], $g\left(\left\|\nabla u_{k}(x, y, t)\right\|\right)$ is defined as

$$
g\left(\left\|\nabla u_{k}(x, y, t)\right\|\right)=e^{-\left(\left\|\nabla u_{k}(x, y, t)\right\| / \sigma\right)^{2}}, \quad k=0, \ldots, 7
$$

or

$$
\begin{array}{r}
g\left(\left\|\nabla u_{k}(x, y, t)\right\|\right)=\frac{1}{1+\left(\left\|\nabla u_{k}(x, y, t)\right\| / \sigma\right)^{2}}, \\
k=0, \ldots, 7,
\end{array}
$$


where $\|\cdot\|$ is the module of the vector, and the constant $\sigma$ controls the sensitivity to edges.

The new FOPMD based on G-L fractional-order derivative is defined as

$$
\frac{\partial u(i, j, t)}{\partial t}=\operatorname{div}^{\alpha}\left(\begin{array}{c}
g_{0} \nabla_{0}^{\alpha} u(i, j, t) \\
g_{1} \nabla_{1}^{\alpha} u(i, j, t) \\
g_{2} \nabla_{2}^{\alpha} u(i, j, t) \\
g_{3} \nabla_{3}^{\alpha} u(i, j, t) \\
g_{4} \nabla_{4}^{\alpha} u(i, j, t) \\
g_{5} \nabla_{5}^{\alpha} u(i, j, t) \\
g_{6} \nabla_{6}^{\alpha} u(i, j, t) \\
g_{7} \nabla_{7}^{\alpha} u(i, j, t)
\end{array}\right),
$$

where $g_{k}, k=0, \ldots, 7$ defined in (11) are the components of $\mathbf{g}$ in (11).

The above equation can be represented as

$$
\frac{\partial u(i, j, t)}{\partial t}=\sum_{k=0}^{7} g_{k} \nabla_{k}^{2 \alpha} u(i, j, t)
$$

where $\sum_{k=0}^{7} g_{k}=1$ and $\nabla_{k}^{2 \alpha} u(i, j, t)$ can be computed according to (9).

Thus the explicit form for solving (16) is

$$
u(i, j, t+1)=u(i, j, t)+\lambda \sum_{k=0}^{7} g_{k} \nabla_{k}^{2 \alpha} u(i, j, t),
$$

where $u(i, j, t+1)$ is the gray level of $(i, j)$ at time $t+1$ and $\lambda$ is the integration constant $(0 \leq \lambda \leq 1 / 7)$. Usually, due to numerical stability, $\lambda$ is set to its maximum value.

To summarize, our sinogram restoration approach is done in the following steps.

(1) Let the input sinogram be $U$ and set $t=1$, $U_{t}=U$, input iterative number $n$, fractional-order $\alpha$, integration constant $\lambda$ in (17), and gradient modulus threshold $\sigma$ that controls the conduction used in (13) or (14) and choose (13) or (14) as diffusion coefficients.

(2) Compute 1-order gradient vector $\nabla \mathbf{u}$ using (7).

(3) Compute diffusion coefficients vector $\mathbf{g}$ using (11)(14).

(4) Compute $2 \alpha$-order gradient vector $\nabla^{2 \alpha} \mathbf{u}$ using (9).

(5) Compute $U_{t+1}$ using (17), and set $t=t+1$, if $t=n$, output sinogram $U_{t}$; else goto step 2 .

(6) Back-project sinogram $U_{t}$ into the image $I$.

\section{Experiments and Discussion}

The main objective for sinogram restoration of LDCT is to delete the noise and avoid artifacts while preserving anatomy details for the back-projection images.
Two abdominal CT images of a 58-year-old man and two abdominal CT images of a 62 -year-old woman with different doses were scanned from a 16 multidetector row CT unit (Somatom sensation 16; Siemens Medical Solutions) using $120 \mathrm{kVp}$ and $5 \mathrm{~mm}$ slice thickness. Other remaining scanning parameters are gantry rotation time, 0.5 second; detector configuration (number of detector rows section thickness), $16 \times 1.5 \mathrm{~mm}$; table feed per gantry rotation, $24 \mathrm{~mm}$; and pitch, $1: 1$ and reconstruction method, back projection (FBP) algorithm with the soft-tissue convolution kernel "B30f". Different CT doses were controlled by using two different fixed tube currents $60 \mathrm{mAs}$ and $150 \mathrm{mAs}(60 \mathrm{~mA}$ or $150 \mathrm{mAs})$ for LDCT and standard-dose CT (SDCT) protocols, respectively. The CT dose index volume (CTDIvol) for LDCT images and SDCT images is in positive linear correlation with the tube current and is calculated to be approximately ranged between 15.32 mGy to 3.16 mGy [27] (see Figures 1(a)-1(d)).

In order to compare our method with classical PM and other state-of-the art FOPMD methods, three compared methods are PMD [1], regularized PMD (RPM) [2], and FOPMD proposed in [9]. Since PMD has been discussed in detail in Section 1. We will briefly introduce other two methods.

It is well known that PMD is an ill-posed equation but RPMD [2] is a well-posed equation, which is defined as

$$
\frac{\partial u(x, y, t)}{\partial t}=\operatorname{div}\left(g\left(\left\|G_{\sigma_{1}} \cdot \nabla u(x, y, t)\right\|\right) \nabla u(x, y, t)\right)
$$

Here $G_{\sigma_{1}}$ is defined as

$$
G_{\sigma_{1}}=\frac{1}{C} e^{-\left(x^{2}+y^{2}\right) / \sigma_{1}^{2}}
$$

is a Gaussian function and $C$ is a constant. The diffusion coefficients $g(\cdot)$ are defined in (13) or (14). That is, the gradient vector in diffusion coefficients is convoluted by a Gaussian kernel to suppressing unsteadiness in image. Both PMD and RPMD use half-point central difference discretization scheme.

The FOPMD proposed in [9] is defined in (4), which is a FSFOD using G-L definition. Both FOPMD in [9] and EFOGV-PMD use integer-point unilateral difference discretization scheme.

In order to ensure that comparison is put on a fair level, the common used parameters are set to the same value. The commonly used parameters for four methods include gradient modulus threshold $\sigma$ that controls the conduction, integration constant $\lambda(0 \leq \lambda \leq 1 / 7)$, and iteration number $t$. Due to numerical stability, $\lambda$ is set to its maximum value $1 / 7$ and $\sigma$ is set to 30 to reduce iteration number.

The iteration number $t$ is very important in PMD. That is, big $t$ will make smooth image while small $t$ will still leave much noise. In order to study the performance of four compared methods with different iteration number $t$ and fixed other parameters, $t$ is set to 3,8 , and 15 , respectively.

The standard deviation of smoothed Gaussian kernel for the image $\sigma_{1}$ used for RPMD is set to 1 since in [2], the authors suggest that $\sigma_{1}$ should be a small number.

On sinogram space, FOPMD with $\alpha=0.5, \alpha=0.8$, and $\alpha=1.2$ is carried on two image collections. 
SDCT image

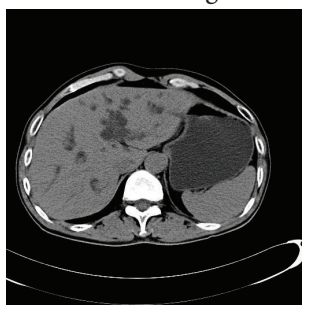

(a) Original SDCT image with tube current time product $150 \mathrm{mAs}$

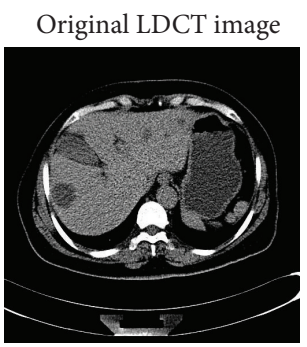

(d) Original LDCT image with tube current time product $60 \mathrm{mAs}$

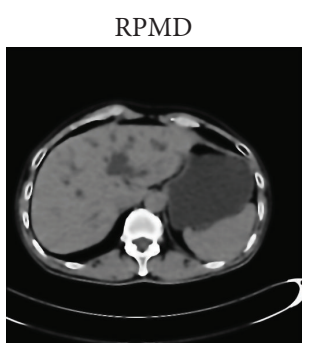

(g) LDCT image (b) processed by RPMD with $\sigma=$ $30, \sigma_{1}=1$, and $\lambda=1 / 7$ and iteration number is 15

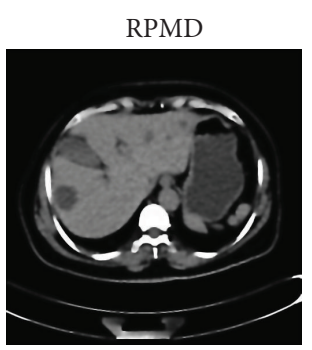

(j) LDCT image (d) processed by RPMD with $\sigma=$ $30, \sigma_{1}=1$, and $\lambda=1 / 7$ and iteration number is 15

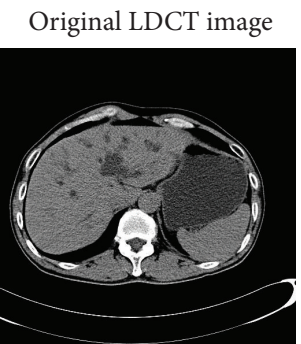

(b) Original LDCT image with tube current time product $30 \mathrm{mAs}$

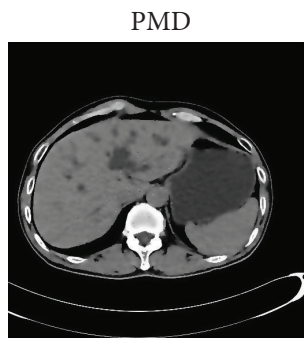

(e) LDCT image (b) processed by PMD with $\sigma=30$, and $\lambda=1 / 7$ and iteration number is 15

FOPMD

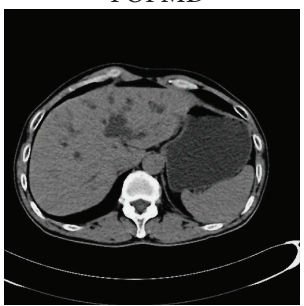

(h) LDCT image (b) processed by FOPMD in [9] with $\sigma=30, \alpha=1$, and $\lambda=$ $1 / 7$ and iteration number is 15

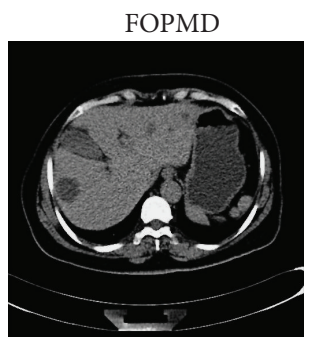

(k) LDCT image (d) processed by FOPMD in [9] with $\sigma=30, \alpha=1$, and $\lambda=$ $1 / 7$ and iteration number is 15
SDCT image

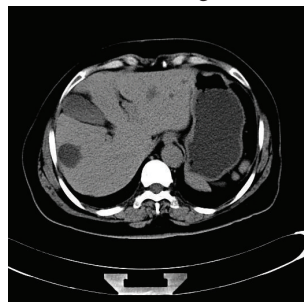

(c) Original SDCT image with tube current time product $150 \mathrm{mAs}$

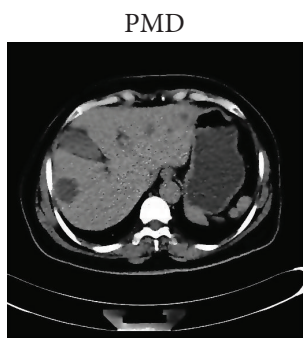

(f) LDCT image (d) processed by PMD with $\sigma=30$, and $\lambda=1 / 7$ and iteration number is 15

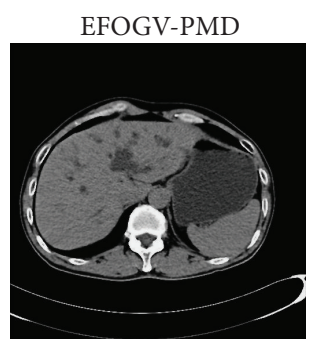

(i) LDCT image (b) processed by EFOGVPMD with $\sigma=30, \alpha=1$ and $\lambda=1 / 7$ and iteration number is 15

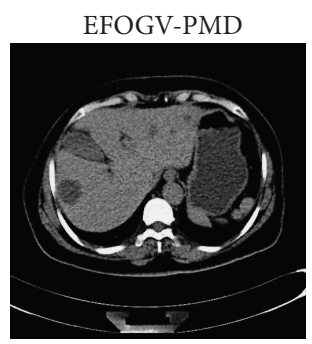

(l) LDCT image (d) processed by EFOGVPMD with $\sigma=30, \alpha=1$, $\lambda=1 / 7$ and iteration number is 15

FIGURE 1: Original SDCT and LDCT images ((a)-(d)), LDCT images processed by PMD, RPMD, FOPMD and EFOGV-PMD with fractionalorder $\alpha=1$ and iteration number is 15 . 
Since bigger iteration number leads to smoother denoised results, in order to observe the behaviors of big iteration number clearly, Figure 1 sets iteration number $t=15$. Comparing all the original SDCT images in Figures 1(a) and 1(c), LDCT images in Figures 1(b) and 1(d) were severely degraded by nonstationary noise. All denoised images in Figure 1 can suppress most of noises. In particular, FOPMD and EFOGVPMD can provide very satisfied images with having little noise and preserving all useful anatomy structures. However, denoised images of PMD and RPMD are oversmooth, which lost a lot of details.

In order to test the consistency of the definitions of different integer order or fractional order, we set fractionalorder $\alpha=1$, in which two fractional-order PMD should have same forms and they also correspond to the order of PMD and RPMD (see Figures 1(e)-1(l)). Observing Figures $1(\mathrm{~h}), 1(\mathrm{i}), 1(\mathrm{k})$, and $1(\mathrm{l})$, we can find that the denoised images are identical, which demonstrate that the fractional-order definitions between [9] and EFOGV-PMD are identical when $\alpha=1$.

However, the resulting images of PMD and RPMD are quite different to the images denoised by FOPMD and EFOGV-PMD. That is, the images processed by PMD and RPMD are smoother than the images processed by FOPMD and EFOGV-PMD. Different discretization schemes, PMD and RPMD adopt half-point central difference while FOPMD and EFOGV-PMD adopt integer-point unilateral difference, leading to this interesting result.

Since two FOMD schemes provide more satisfying results, we only compare two FOPMD methods with different fractional orders (see Figure 2) and different iteration numbers (see Figure 3).

In order to compare denoised results of two FOPMD schemes with different fractional orders, two original LDCT images in Figures 1(b) and 1(d) are used with iteration number $t=15$ and fractional order $\alpha=0.5, \alpha=0.8$ and $\alpha=1.2$. From the second and the forth rows of Figure 2, we can conclude that the resulting images of EFOGV-PMD are very satisfied and they become smoother when $\alpha$ becomes bigger, which is coherence with our intuition.

However, denoised images Figures 2(a) and 2(g) with $\alpha=$ 0.5 using FOPMD in [9] have many artifacts, which are small black circles in two images. In addition, denoised images Figures 2(c) and 2(i) are very dark compared with the original LDCT images in Figures 1(b) and 1(d). Images in Figures 2(c) and 2(i) also have some isolated artificial white points, which are called speckle effect. It is obvious that resulting images in Figures 2(b) and 2(h) with $\alpha=0.8$ processed by FOPMD in [9] have the best performance in three image series with different $\alpha$.

Generally, artifacts in denoised images are oscillations near edges, caused by that the low-passed filtering is not processed correctly near the real edges. That is, some smooth regions near edges are regarded false as edges, which make these error edges preserved. Therefore, improving accuracy of edge detection is a good choice for improving the performance of FOPMD in [9]. This is the start point of our new method proposed in this paper.
From Figure 1, we can see the processed images using FOPMD in [9] with $\alpha=1$ are very satisfied, which implies that when $\alpha=1$, FOPMD in [9] can locate edges correctly. In addition, processed images with $\alpha=0.8$, whose fractional orders are closest to $\alpha=1$ in three image series, also are best in the three image series. In another words, the processed images with nearer fractional order to 1 have better performance. Therefore, $\alpha=1$ is the best choice for edge locator.

Although when $\alpha=1$, the FOPMD in [9] has the best performance for edge detection, impressive characters for fractional-order PMD with well preserved edges and power of suppressing noises are also promising in sinogram restoration of LDCT. Thus we adopt a new scheme to get both advantages of integer-order and fraction-order PMD. That is, the gradient vector in diffusion coefficients is integer-order to ensure correct edge detection while the "external" gradient vector is fractional order to suppressing noise and preserving edges.

Intuitively, processed images with bigger iteration number correspond to smoother images. In order to check the influence of iteration numbers for FOPMD in [9] and EFOGV-PMD, processed image series of two original LDCT images in Figures 1(b) and 1(d) with different iteration numbers 3, 8 and 15 are shown in Figure 3.

The resulting images in the first and the third rows of Figure 3 are the processed images using FOPMD in [9]. Compared with original LDCT images in Figures 1(b) and $1(\mathrm{~d})$, all resulting images with different iteration numbers have less noise. In addition, the smoother images can be obtained as the iteration number becomes bigger. However, the most undesired default for FOPMD in [9] is that resulting images become dark as the iteration number becomes big. Moreover, except for Figure 3(g), the resulting images in the first and the third rows have some isolated white points, which are the speckle effect.

The resulting images in the second and the forth rows of Figure 3 show that EFOGV-PMD with different iteration numbers are very satisfied and they become smoother when the iteration number becomes bigger, which is in coherence with our intuition. Another attractive nature for EFOGVPMD about iteration is that the smoothing shown in these images is very slow. That is, the resulting images in Figures 3(d) and 3(j) with iteration number 3 are slightly different to the images in Figures 3(f) and 3(l) with iteration number 15. This nature shows that EFOGV-PMD has good stability. Therefore, it is not sensitive to iteration number.

Recall that FOPMD in [9] makes artifacts, dark resulting images, and speckle effects. It should be indicated that although FOPMD in [9] suffers from above disadvantages, it can obtain satisfied resulting images by choosing suitable parameters. In addition, all exiting FOPMD methods at least suffer from speckle effect from the resulting images of these images. Fortunately, EFOGV-PMD can avoid artifacts, dark images, and speckle effect completely, which ensure its applications in sinogram restoration. More important for the new scheme is its stability, which makes it not sensitive to the iteration number. 
FOPMD

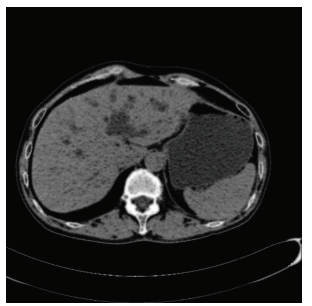

(a) LDCT image in Figure 1(b) processed by FOPMD in [9] with $\sigma=30, \alpha=0.5$, and $\lambda=1 / 7$ and iteration number is 15

EFOGV-PMD

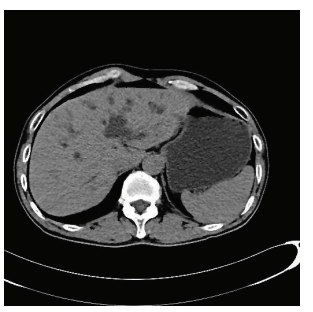

(d) LDCT image in Figure 1(b) processed by EFOGV-PMD with $\sigma=30, \alpha=0.5$, and $\lambda=1 / 7$ and iteration number is 15

FOPMD

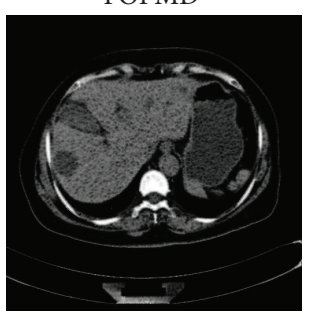

(g) LDCT image in Figure 1(d) processed by FOPMD in [9] with $\sigma=30, \alpha=0.5$, and $\lambda=1 / 7$ and iteration number is 15

EFOGV-PMD

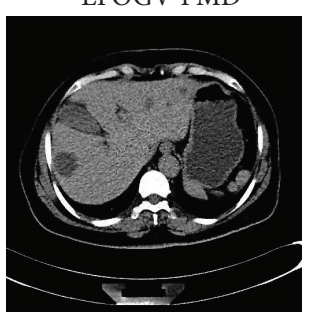

(j) LDCT image in Figure 1(d) processed by EFOGV-PMD with $\sigma=30, \alpha=0.5$, and $\lambda=1 / 7$ and iteration number is 15
FOPMD

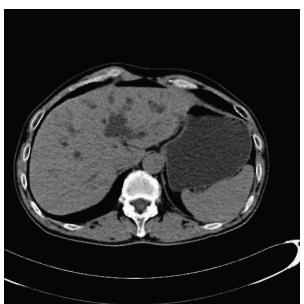

(b) LDCT image in Figure 1(b) processed by FOPMD in [9] with $\sigma=30, \alpha=0.8$, and $\lambda=1 / 7$ and iteration number is 15

EFOGV-PMD

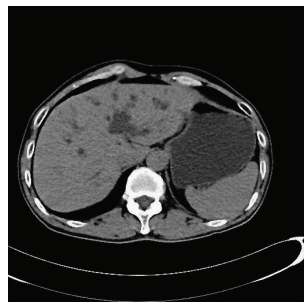

(e) LDCT image in Figure 1(b) processed by EFOGV-PMD with $\sigma=30, \alpha=0.8$, and $\lambda=1 / 7$ and iteration number is 15

FOPMD

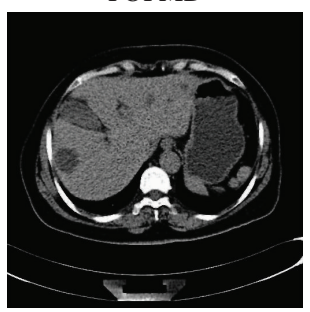

(h) LDCT image in

Figure 1(d) processed by FOPMD in [9] with $\sigma=30, \alpha=0.8$, and $\lambda=1 / 7$ and iteration number is 15

EFOGV-PMD

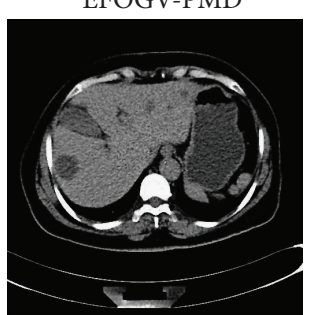

(k) LDCT image in Figure 1(d) processed by EFOGV-PMD with $\sigma=30, \alpha=0.8$, and $\lambda=1 / 7$ and iteration number is 15
FOPMD

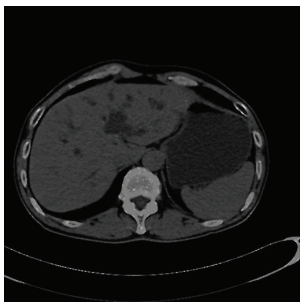

(c) LDCT image in Figure 1(b) processed by FOPMD in [9] with $\sigma=30, \alpha=1.2$, and $\lambda=1 / 7$ and iteration number is 15

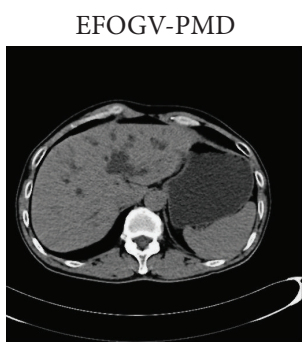

(f) LDCT image in Figure 1(b) processed by EFOGV-PMD with $\sigma=30, \alpha=1.2$, and $\lambda=1 / 7$ and iteration number is 15

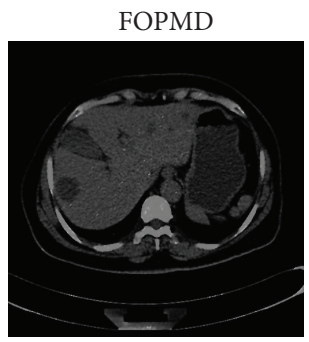

(i) LDCT image in Figure 1(d) processed by FOPMD in [9] with $\sigma=30, \alpha=1.2$, and $\lambda=1 / 7$ and iteration number is 15

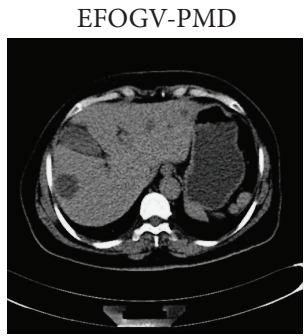

(l) LDCT image in Figure 1(d) processed by EFOGV-PMD with $\sigma=30, \alpha=1.2$, and $\lambda=1 / 7$ and iteration number is 15

FIGURE 2: LDCT images in Figures 1(b) and 1(d) processed by FOPMD and EFOGV-PMD with different fractional-orders and iteration number is set to 15 . The first column: $\alpha=0.5$; the second column: $\alpha=0.8$, the third column: $\alpha=1.2$. The first row: Figure 1(b) processed by FOPMD; the second row: Figure 1(b) processed by EFOGV-PMD; the third row: Figure 1(d) processed by FOPMD; the fourth row: Figure 1(d) processed by EFOGV-PMD. 
FOPMD

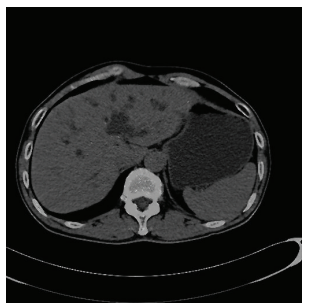

(a) LDCT image in Figure 1(b) processed by FOPMD in [9] with $\sigma=30, \alpha=1.2$, and $\lambda=1 / 7$ and iteration number is 3

EFOGV-PMD

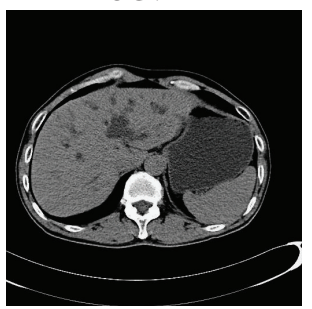

(d) LDCT image in Figure 1(b) processed by EFOGV-PMD with $\sigma=30, \alpha=1.2$, and $\lambda=1 / 7$ and iteration number is 3

FOPMD

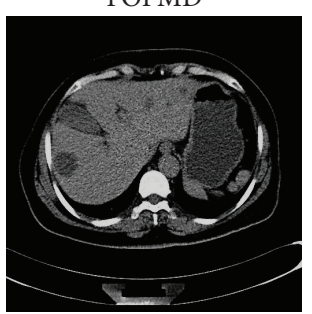

(g) LDCT image in Figure 1(d) processed by FOPMD in [9] with $\sigma=30, \alpha=1.2$, and $\lambda=1 / 7$ and iteration number is 3

EFOGV-PMD

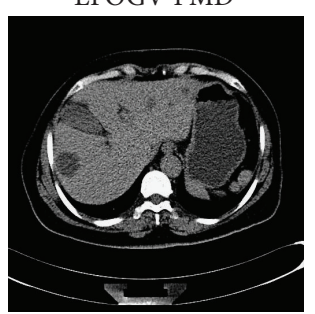

(j) LDCT image in Figure 1(d) processed by EFOGV-PMD with $\sigma=30, \alpha=1.2$, and $\lambda=1 / 7$ and iteration number is 3
FOPMD

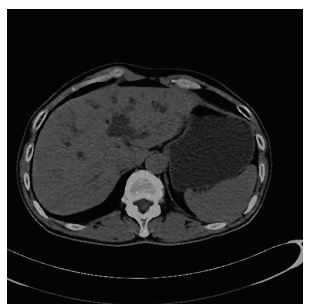

(b) LDCT image in Figure 1(b) processed by FOPMD in [9] with $\sigma=30, \alpha=1.2$, and $\lambda=1 / 7$ and iteration number is 8

EFOGV-PMD

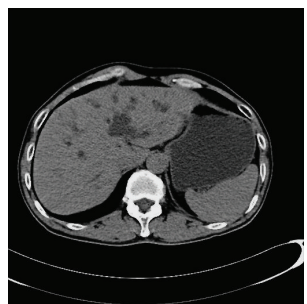

(e) LDCT image in Figure 1(b) processed by EFOGV-PMD with $\sigma=30, \alpha=1.2$, and $\lambda=1 / 7$ and iteration number is 8

FOPMD

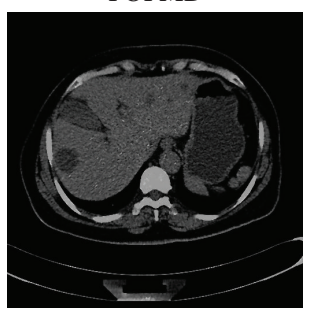

(h) LDCT image in

Figure 1(d) processed by FOPMD in [9] with $\sigma=30, \alpha=1.2$, and $\lambda=1 / 7$ and iteration number is 8

EFOGV-PMD

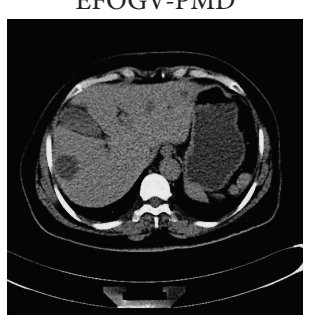

(k) LDCT image in Figure 1(d) processed by EFOGV-PMD with $\sigma=30, \alpha=1.2$, and $\lambda=1 / 7$ and iteration number is 8
FOPMD

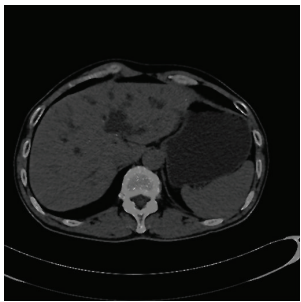

(c) LDCT image in Figure 1(b) processed by FOPMD in [9] with $\sigma=30, \alpha=1.2$, and $\lambda=1 / 7$ and iteration number is 15

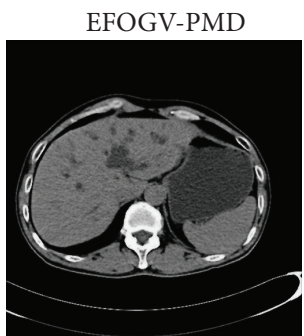

(f) LDCT image in Figure 1(b) processed by EFOGV-PMD with $\sigma=30, \alpha=1.2$, and $\lambda=1 / 7$ and iteration number is 15

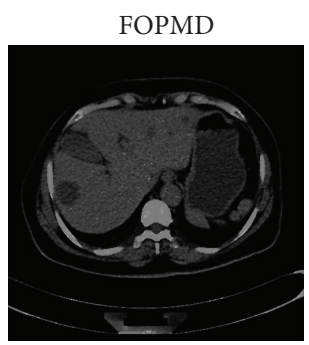

(i) LDCT image in Figure 1(d) processed by FOPMD in [9] with $\sigma=30, \alpha=1.2$, and $\lambda=1 / 7$ and iteration number is 15

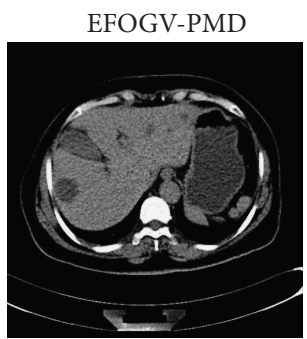

(1) LDCT image in Figure 1(d) processed by EFOGV-PMD with $\sigma=30, \alpha=1.2$, and $\lambda=1 / 7$ and iteration number is 15

FIGURE 3: LDCT images in Figures 1(b) and 1(d) processed by FOPMD and EFOGV-PMD with different iteration numbers and the fractionalorder $\alpha=1.2$. The first column: iteration number is 3 ; the second column: iteration number is 8 and the third column: iteration number is 15 . The first row: Figure 1(b) processed by FOPMD; the second row: Figure 1(b) processed by EFOGV-PMD; the third row: Figure 1(d) processed by FOPMD and the fourth row: Figure 1(d) processed by EFOGV-PMD. 


\section{Conclusions}

In this paper, we propose a new FOPMD, EFOGV-PMD for LDCT sinogram imaging based on G-L fractional-order derivative definition. EFOGV-PMD not only has good ability to preserving edges while denoising, it also can avoid artifacts, dark images and speckle effects of FOPMD in [9] and other existing FOPMD schemes completely by correctly located edges, which ensures that EFOGV-PMD can be used for sinogram restoration of LDCT. More importantly, EFOGV-PMD has good stability for iteration numbers, which leads to it is not sensitive to the iteration number choice. Having so many advantages for EFOGV-PMD, it should become an promising candidate method for sinogram restoration of LDCT.

\section{Acknowledgments}

This paper is supported by the National Natural Science Foundation of China (no 60873102), the Major State Basic Research Development Program (no. 2010CB732501), and the Open Foundation of Visual Computing and Virtual Reality Key Laboratory Of Sichuan Province (no. J2010N03). This work was supported by a Grant from the National High Technology Research and Development Program of China (no. 2009AA12Z140).

\section{References}

[1] P. Perona and J. Malik, "Scale-space and edge detection using anisotropic diffusion," IEEE Transactions on Pattern Analysis and Machine Intelligence, vol. 12, no. 7, pp. 629-639, 1990.

[2] F. Catté, P.-L. Lions, J.-M. Morel, and T. Coll, "Image selective smoothing and edge detection by nonlinear diffusion," SIAM Journal on Numerical Analysis, vol. 29, no. 1, pp. 182-193, 1992.

[3] Y.-L. You and M. Kaveh, "Fourth-order partial differential equations for noise removal," IEEE Transactions on Image Processing, vol. 9, no. 10, pp. 1723-1730, 2000.

[4] T. F. Chan, S. Esedoglu, and F. E. Park, "A fourth order dual method for staircase reduction in texture extraction and image restoration problems," http://basilo.kaist.ac.kr/preprint/uclam/cam05-28.pdf.

[5] T. Chan, A. Marquina, and P. Mulet, "High-order total variation-based image restoration," SIAM Journal on Scientific Computing, vol. 22, no. 2, pp. 503-516, 2000.

[6] J. Bai and X.-C. Feng, "Fractional-order anisotropic diffusion for image denoising," IEEE Transactions on Image Processing, vol. 16, no. 10, pp. 2492-2502, 2007.

[7] J. Zhang and Z. Wei, "A class of fractional-order multi-scale variational models and alternating projection algorithm for image denoising," Applied Mathematical Modelling, vol. 35, no. 5, pp. 2516-2528, 2011.

[8] M. Janev, S. Pilipović, T. Atanacković, R. Obradović, and N. Ralević, "Fully fractional anisotropic diffusion for image denoising," Mathematical and Computer Modelling, vol. 54, no. 1-2, pp. 729-741, 2011.

[9] S. Hu, Z. Liao, and W. Chen, "Sinogram restoration for lowdosed X-ray computed tomography using fractional-order Perona-Malik diffusion," Mathematical Problems in Engineering, vol. 2012, Article ID 391050, 13 pages, 2012.
[10] M. Li, "Approximating ideal filters by systems of fractional order," Computational and Mathematical Methods in Medicine, vol. 2012, Article ID 365054, 6 pages, 2012.

[11] M. Li, S. C. Lim, C. Cattani, and M. Scalia, "Characteristic roots of a class of fractional oscillators," Advances in High Energy Physics, vol. 2013, Article ID 853925, 2013.

[12] M. Li and W. Zhao, "On 1/f noise," Mathematical Problems in Engineering, vol. 2012, Article ID 673648, 23 pages, 2012.

[13] M. Li and W. Zhao, "Solving Abel's type integral equation with Mikusinski's operator of fractional order," Advances in Mathematical Physics, vol. 2013, Article ID 806984, 4 pages, 2013.

[14] D. J. Brenner and E. J. Hall, "Computed tomography-an increasing source of radiation exposure," The New England Journal of Medicine, vol. 357, no. 22, pp. 2277-2284, 2007.

[15] L. Yu, "Radiation dose reduction in computed tomography: techniques and future perspective," Imaging in Medicine, vol. 1, no. 1, pp. 65-84, 2009.

[16] J. Weidemann, G. Stamm, M. Galanski, and M. Keberle, "Comparison of the image quality of various fixed and dose modulated protocols for soft tissue neck CT on a GE Lightspeed scanner," European Journal of Radiology, vol. 69, no. 3, pp. 473477, 2009.

[17] M. K. Kalra, M. M. Maher, M. A. Blake et al., "Detection and characterization of lesions on low-radiation-dose abdominal CT images postprocessed with noise reduction filters," Radiology, vol. 232, no. 3, pp. 791-797, 2004.

[18] J. C. R. Giraldo, Z. S. Kelm, L. S. Guimaraes et al., "Comparative study of two image space noise reduction methods for computed tomography: bilateral filter and nonlocal means," Proceedings of the Annual International Conference of the IEEE Engineering in Medicine and Biology Society, vol. 1, pp. 35293532,2009

[19] A. Manduca, L. Yu, J. D. Trzasko et al., "Projection space denoising with bilateral filtering and CT noise modeling for dose reduction in CT,' Medical Physics, vol. 36, no. 11, pp. 49114919, 2009

[20] N. Mail, D. J. Moseley, J. H. Siewerdsen, and D. A. Jaffray, "The influence of bowtie filtration on cone-beam CT image quality," Medical Physics, vol. 36, no. 1, pp. 22-32, 2009.

[21] Z. Liao, S. Hu, and W. Chen, "Determining neighborhoods of image pixels automatically for adaptive image denoising using nonlinear time series analysis," Mathematical Problems in Engineering, vol. 2010, Article ID 914564, 14 pages, 2010.

[22] H. Lu, I.-T. Hsiao, X. Li, and Z. Liang, "Noise properties of low-dose CT projections and noise treatment by scale transformations," in Proceedings of IEEE Nuclear Science Symposium Conference Record, vol. 3, pp. 1662-1666, November 2001.

[23] J. Xu and B. M. W. Tsui, "Electronic noise modeling in statistical iterative reconstruction," IEEE Transactions on Image Processing, vol. 18, no. 6, pp. 1228-1238, 2009.

[24] I. A. Elbakri and J. A. Fessler, "Statistical image reconstruction for polyenergetic X-ray computed tomography," IEEE Transactions on Medical Imaging, vol. 21, no. 2, pp. 89-99, 2002.

[25] J. Wang, H. Lu, J. Wen, and Z. Liang, "Multiscale penalized weighted least-squares sinogram restoration for low-dose Xray computed tomography," IEEE Transactions on Biomedical Engineering, vol. 55, no. 3, pp. 1022-1031, 2008.

[26] J. Wang, T. Li, H. Lu, and Z. Liang, "Penalized weighted least-squares approach to sinogram noise reduction and image reconstruction for low-dose X-ray computed tomography," 
IEEE Transactions on Medical Imaging, vol. 25, no. 10, pp. 12721283, 2006.

[27] Y. Chen, W. Chen, X. Yin et al., "Improving low-dose abdominal CT images by weighted intensity averaging over large-scale neighborhoods," European Journal of Radiology, vol. 80, no. 2, pp. e42-e49, 2011. 


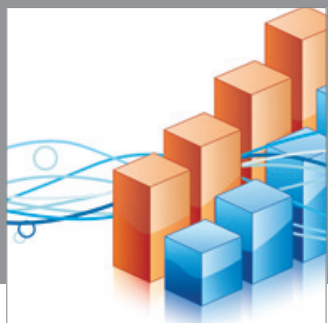

Advances in

Operations Research

mansans

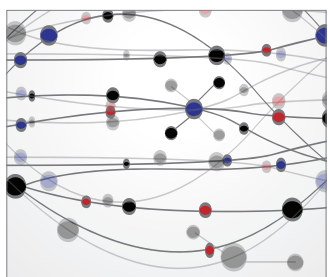

The Scientific World Journal
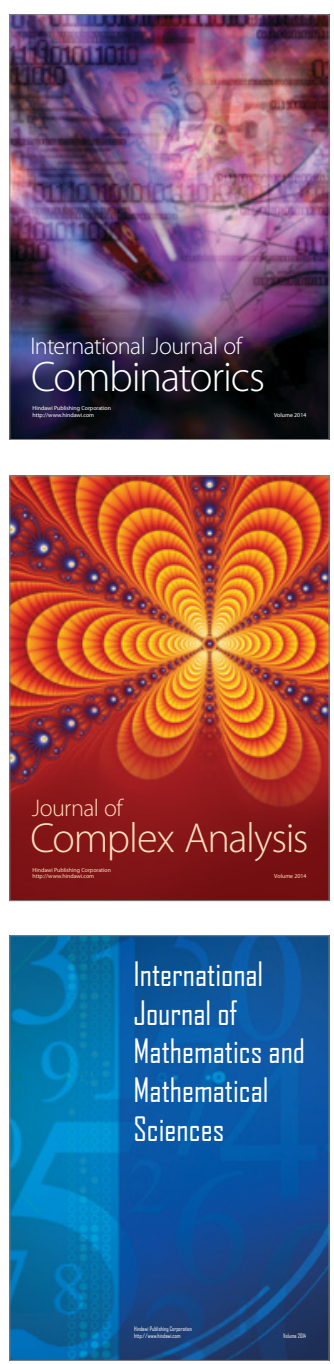
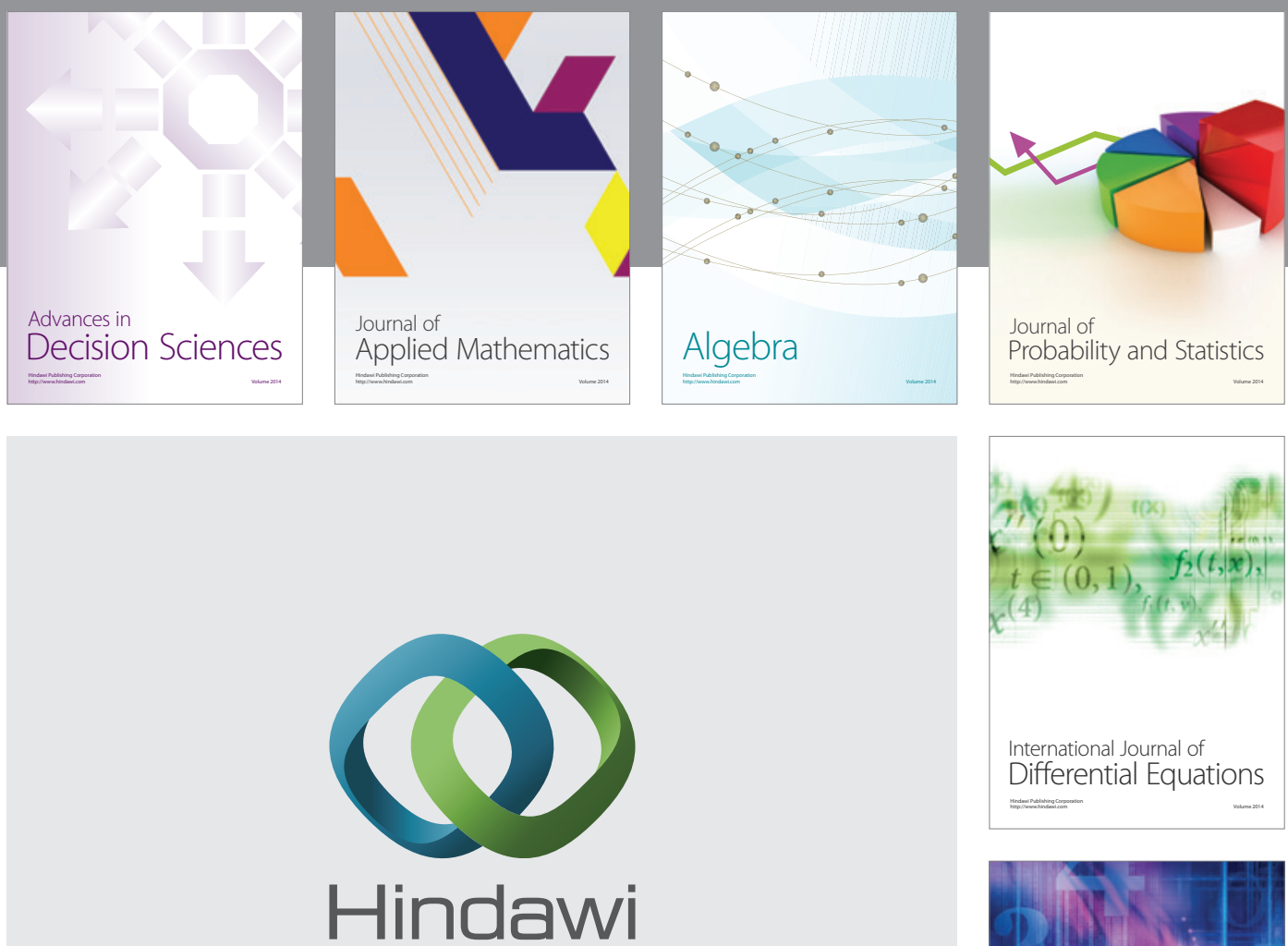

Submit your manuscripts at http://www.hindawi.com
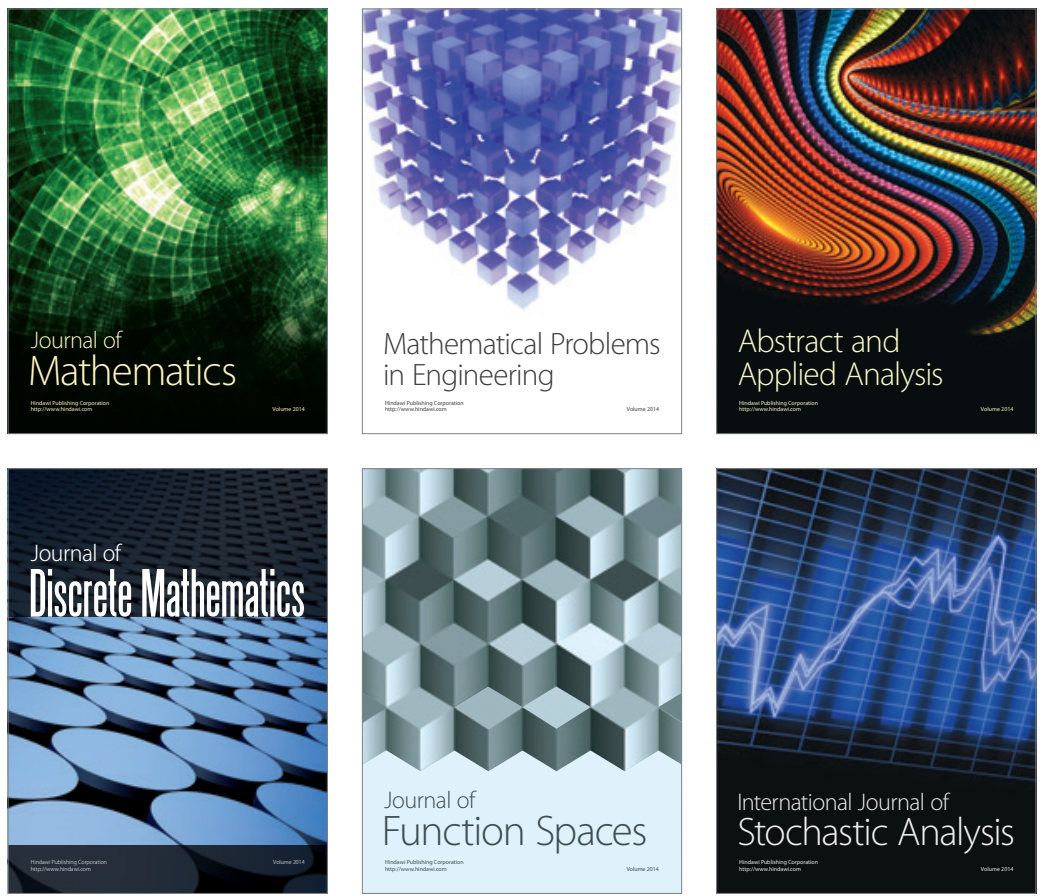

Journal of

Function Spaces

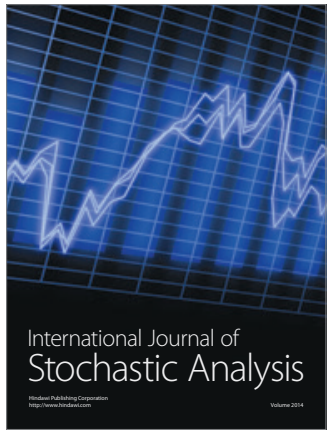

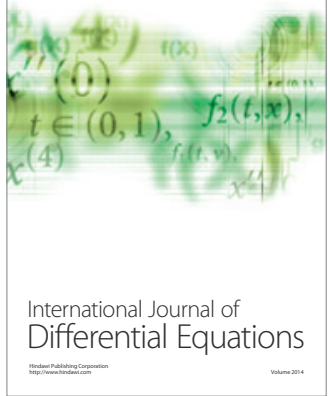
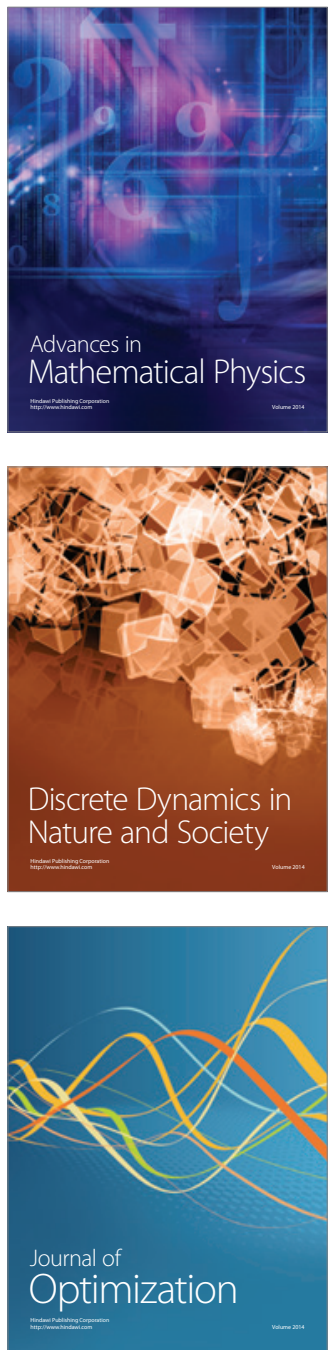